\title{
Clinical protocol for a high insertion torque immediate implant in fresh extraction sites: A 3-year audit
}

\author{
Tak-Kun Chow* \\ Specialist in Oral \& Maxillofacial Surgery, Suite 1322, Central Building, 1-3 Pedder Street, Central, Hong Kong
}

\begin{abstract}
Purpose: The purpose of this report was to evaluate the clinical protocol of a variable-thread tapered implants placed by high insertion torque in fresh extraction sites either loaded immediately or in stages in a 3-year period. The deciding parameters including insertion torque, implant dimension, extraction socket status, jumping gap grafting decision, time to definitive restoration loading and the cumulative survival rate were analytically evaluated.

Materials \& methods: 45 implants were placed in 34 patients in a private oral maxillofacial surgery specialist practice according to a standard protocol. All teeth were removed a traumatically then immediate implant placement into the fresh extraction socket that either restored provisionally or in staged protocol. For those immediate temporized and function implants were installed via flapless approach except at molar region. Peri-implant gap to extraction socket wall $>1.5 \mathrm{~mm}$ were grafted. Definitive restorations all were placed within the first year. Regular Clinical and radiographic assessment were performed after implant placement at 6 months, 12 months and 24 months.

Results: Thirty-four patients were treated and were restored with 41 single crowns and 2 two-unit bridges. Amongst 45 implants, 27 were immediately temporary restored and function were all survive. Contrary, two one-staged immediate implants were failed need redo prior to 3 months. Therefore the overall cumulative survival rate in this cohort became 95.6\%. Male to Female ratio $=16: 18$ with the mean age was $50.6 \pm 13.5$ (range 17-76). The distribution of implant placement found mainly in maxilla $84.4 \%$ (38 implants). Ten teeth $(22.2 \%)$ presented with peri-apical abscess prior to surgery that submitted to immediate implant surgery without any case of failure in this series. High insertion torque $>50-70 \mathrm{Ncm}$ could be achieved in $73.3 \%(33 / 45)$ of implants as its initial stability with medium bone quality as majority (84.4\%). 64\% peri-implant gap $>1.5 \mathrm{~mm}$ were grafted so as to maintain an optimal peri-implant bone healing. Regarding implant dimension, almost $31.1 \%$ were RP $5 \mathrm{mmx} 13 \mathrm{~mm}$ (14 out of 45 ) being selected most commonly for immediate implant placement. The mean timing for final prosthetic loading were $3.4 \pm 1.2$ months (range 3 weeks to 8 months)
\end{abstract}

Conclusion: The 3-Year results show that immediate placement of a variable-thread tapered implants by high insertion torque $(\geq 50-70 \mathrm{Ncm})$ in fresh extraction socket could achieve a high survival rate at $95.6 \%$, moreover immediate temporary restored and function group even achieve $100 \%$ cumulative survival in this cohort. Several pivotal parameters were concluded as a clinical protocol for immediate post-extraction implant rehabilitation.

\section{Introduction}

The outcome of conventional staged protocol for dental implant osseointegration has been shown to be highly predictable and successful regardless many different designs and brands of implants [1-3]. Inevitability, earliest implant rehabilitation could relieve both psychosocial and oral dental functional impact to patient after tooth lost that should be the parameter of care in contemporary implant dentistry.

Immediate implant placement in fresh extraction socket is a technique that has been developed, especially within the last decade, although it could be dated back to late 1970s at the University of Tubingen, an implant model was specially designed for such procedure [4]. In recent years, there has been an increase in the number of studies on immediate placement which present their results a high success rate of osseointegration as comparable to conventional staged approach [5]. In order to secure a predictable outcome, the clinician should well master the bio-physiology of fresh extraction socket healing in addition to the initial stability of implant placement. Primary or initial Implant stability is of paramount important for implant placement in fresh extraction sockets and immediately loaded [6]. In 2007, a variablethread implant (Nobel Active ${ }^{\mathrm{TM}}$ ) with it's feature of high insertion torque [7] enhancing immediate implant placement was introduced.
Other notable features to enhance immediate implant placement [8]: the TiUnite rough-surfaced, macroscopic grooves placed on the implant threads, differential width of implant body with apical drilling blades and the back-tapered coronal design (Figure 1).

The purpose of report was to evaluate a clinical protocol of this novel implant when used for immediate placement in fresh extraction sockets by an evidence based approach. Those dependent parameters including insertion torque, implant dimension, extraction socket status, jumping gap grafting decision, time to definitive restoration and the cumulative survival rates were evaluated over a three-year period.

\section{Materials and methods}

45 Nobel Active implants were placed in 34 consecutive patients in one private oral maxillofacial surgery specialist practice according to a standard protocol from March 2009 to July 2012. Dental status all

${ }^{\star}$ Correspondence to: Tak-Kun Chow, Specialist in Oral \& Maxillofacial Surgery, Suite 1322, Central Building, 1-3 Pedder Street, Central, Hong Kong, Tel: (852) 2525 0905, E-mail: chowtkomfs@gmail.com

Received: November 07, 2018; Accepted: November 19, 2018; Published: November 21, 2018 


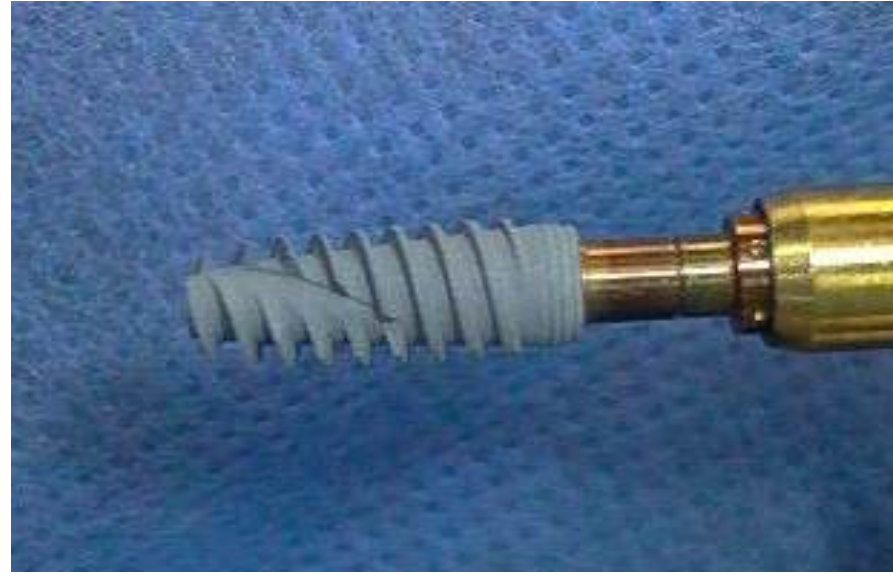

Figure 1. NobelActive ${ }^{\mathrm{TM}}$ implant

were fully assessed by digital Panoramic X-ray (Figure 2) and most of cases further evaluated by dental CT. Anatomical appraisal for those tooth with peri-apical pathology causing labial bone destruction or close in vicinity to vital structure such as inferior dental nerve that could precisely anticipated by 3D CT image reconstruction. All teeth were removed a traumatically then immediate implant placement into the fresh extraction socket (Figure3) that either restored provisionally (Figure 4) or in staged protocol. For those immediate restored and function implants were installed via flapless approach except at molar region. All implants were installed at least $3 \mathrm{~mm}$ apical to gingival sulcus and aiming at $3 \mathrm{~mm}$ bony engagement at the socket apex then final tightening to attain the torque value at least $>35 \mathrm{Ncm}$ for immediate function situation. The occlusal scheme of temporary crown was put in a mutual protected occlusion so as to minimize non-axial forces. Peri-implant gap to extraction socket wall $>1.5 \mathrm{~mm}$ were grafted by nano $\beta$-phase tri-calcium phosphate (Synthograft ${ }^{\mathrm{TM}}$ ) then further secured by a peri-gingival suturing. All immediate restored group were scheduled a bi-weekly follow-up appointment to ensure firm and stable implant function in first month post-operatively. Definitive restorations all were placed within the first year. Regular Clinical and radiographic assessment were performed after implant placement at 6 months, 12 months and 24 months. Peri-implant gingival stability, crestal bone level maintenance are the key monitoring parameters (Figure 5). Regarding the patient's medical status, four of them were chronic smoker, 2 patients with stable Diabetes, 1 patient with Ischaemic Heart Disease, 2 patients with previous history of colonic cancer with remission, 1 patient on Efexor (Serotonin-norepinephrine reuptake inhibitors) for management of depression. Only one patient in each medical condition (stable diabetes, old colonic cancer without chemotherapy and chronic smoker) were selected for immediate placement, temporization and function.

\section{Results}

Thirty-four patients were treated and were restored with 41 single crowns and 2 two-abutments unit bridges. Male to Female ratio = 16:18 with the mean age was $50.6 \pm 13.5$ (range 17-76). Majority of patients suffered from Crown \& Bridge work failure 31.1\% (14/45) (Table 1) that sought for dental implant rehabilitation. The distribution of implant placement found mainly in maxilla $84.4 \%$ (38 implants). Moreover, the most common area for implant placement were the maxilla central and lateral incisors (33.3\% and $15.6 \%$ respectively). Ten teeth $(22.2 \%)$ presented with peri-apical or periodontal abscess prior to surgery that submitted to immediate implant without any case of failure in this series (Table 2). Maximum torque $(\mathrm{Ncm})$ to achieve final tightening of implant as initial stability (Table 3 ) of implant were found to be $\geq 70(55.6 \%), \geq 50(17.8 \%)$ and $\geq 35(24.4 \%)$ and $<35(2.2 \%)$. Medium bone (Type II \& III bone) quality for bone drilling was found to be the majority $(84.4 \%)$ in this series whilst remaining were soft bone (Type IV bone) quality (Table 4). The implant-socket discrepancy was quantified by peri-implant gap $>1.5 \mathrm{~mm}$ ( $64 \%$ of cases) that need Synthograft $^{\mathrm{TM}}$ (nano $\beta$-phase tri-calcium phosphate) packing so as to maintain optimal peri-implant bone healing. Regular platform implant of diameter $5 \mathrm{~mm} \times 13 \mathrm{~mm}$ length was the most commonly selected dimension (Table 5) for immediate placement 31.1\% (14/45). The final prosthetic loading time for staged immediate implants (18/45) was $3.6 \pm 1.1$ months (range 1 to 6 months) (Table 4) though the overall definitive restoration time was $3.4+1.2$ months (range 3 weeks to 8

Table 1. Reasons of tooth extraction

\begin{tabular}{|l|c|}
\hline Indication of tooth extraction & Number (\%) \\
\hline Crown\& Bridge Failures (including post-crown failure) & $14(31.1 \%)$ \\
\hline Crown fracture & $9(20 \%)$ \\
\hline Advanced periodontal disease & $7(15.6 \%)$ \\
\hline Gross caries tooth & $5(11.1 \%)$ \\
\hline Non-salvable RCT tooth & $4(8.9 \%)$ \\
\hline Non-salvable crack tooth & $4(8.9 \%)$ \\
\hline Ankylosed tooth & $2(4.4 \%)$ \\
\hline Total & $\begin{array}{c}\mathbf{4 5} \text { (10 teeth with peri- } \\
\text { apical abscess) }\end{array}$ \\
\hline
\end{tabular}

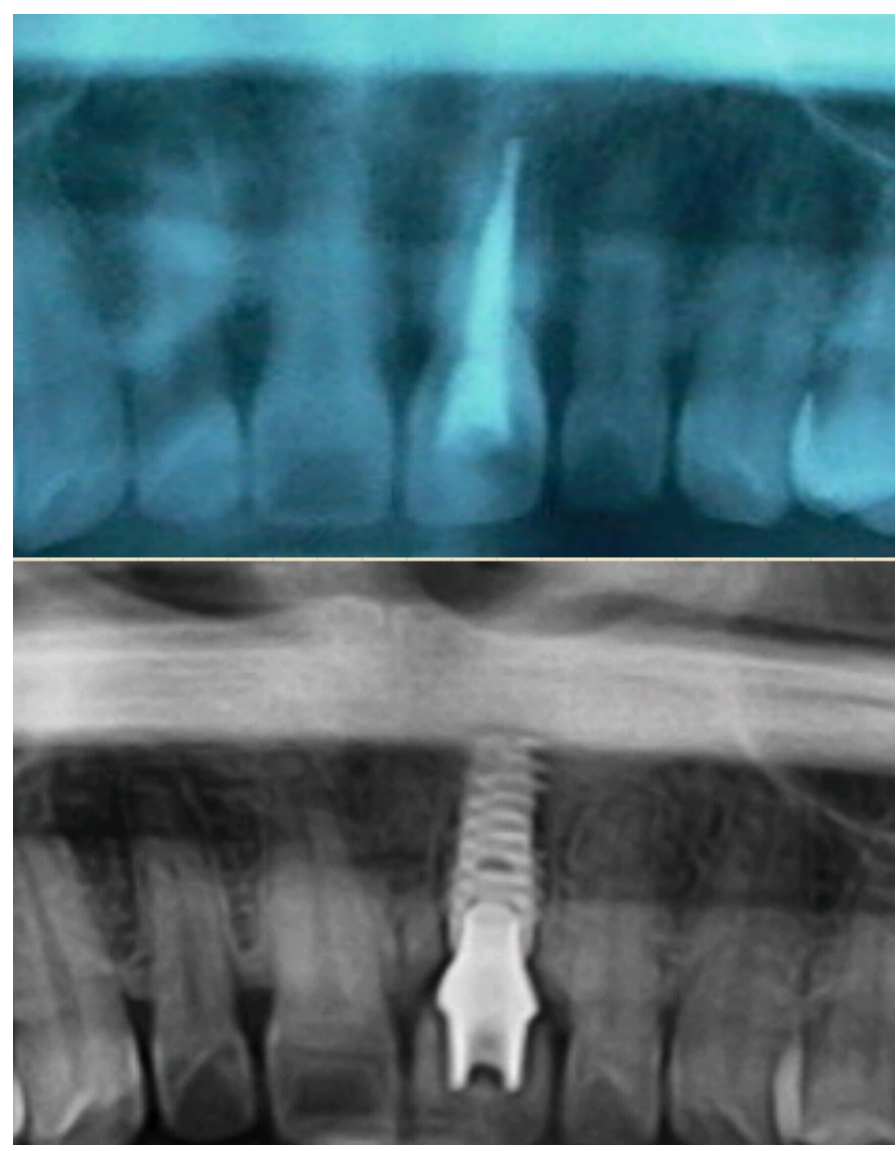

Figure 2. Top: Root treated 21 crown with subgingival fracture, Bottom: Immediate implant placement, temporization and function after 2.5years, X-ray showed excellent bone level maintenance at implant collar 
Table 2. Tooth positioning for forty-five immediate implant placement

\begin{tabular}{|c|c|c|}
\hline Tooth & Maxilla Number (\%) & Mandible Number (\%) \\
\hline Central Incisor & $\mathbf{1 5}(\mathbf{3 3 . 3} \%)$ & $2(4.4 \%)$ \\
\hline Lateral Incisor & $7(15.6 \%)$ & 0 \\
\hline Canine & $3(6.7 \%)$ & $1(2.2 \%)$ \\
\hline First premolar & $6(13.3 \%)$ & $1(2.2 \%)$ \\
\hline Second premolar & $4(8.9 \%)$ & 0 \\
\hline First molar & $1(2.2 \%)$ & $2(4.4 \%)$ \\
\hline Second molar & $2(4.4 \%)$ & $1(2.2 \%)$ \\
\hline Total & $\mathbf{3 8}(\mathbf{8 4 . 4 \% )}$ & $\mathbf{7 ( 1 5 . 4 \% )}$ \\
\hline
\end{tabular}

Table 3. Torque value for final tightening of immediate implant insertion

\begin{tabular}{|c|c|c|c|c|}
\hline Torque (Ncm) & $<\mathbf{3 5}$ & $\mathbf{2 3 5}$ & $\geq \mathbf{5 0}$ & $\geq \mathbf{7 0}$ \\
\hline Maxilla & 1 & 9 & 6 & 22 \\
\hline Mandible & 0 & 2 & 2 & 3 \\
\hline Overall & $\mathbf{1 ( 2 . 2 \% )}$ & $\mathbf{1 1}(\mathbf{2 4 . 4 \% )}$ & $\mathbf{8 ( 1 7 . 8 \% )}$ & $\mathbf{2 5}(\mathbf{5 5 . 4 \% )}$ \\
\hline
\end{tabular}

Table 4. Implantation parameters for immediate placement

\begin{tabular}{|l|c|c|}
\hline Parameters & Immediate function & $\begin{array}{c}\text { Staged protocol (Failed } \\
\text { implants) }\end{array}$ \\
\hline Bone quality- Soft & 0 & $7(1)$ \\
\hline Medium & 27 & $11(1)$ \\
\hline $\begin{array}{l}\text { Torque value } \text { at final } \\
\text { tightening to achieve initial } \\
\text { stability } \\
<35 \mathrm{Ncm}\end{array}$ & 0 & 1 \\
\hline$\geq 35 \mathrm{Ncm}$ & 5 & 6 \\
\hline$\geq 50 \mathrm{Ncm}$ & 6 & 1 \\
\hline$\geq 70 \mathrm{Ncm}$ & 16 & 10 \\
\hline Prosthetic rehabilitation & $\begin{array}{c}\text { Immediate temporized and } \\
\text { function }\end{array}$ & $\begin{array}{c}3.6 \pm 1.1 \text { months (range } 1 \\
\text { month to } 6 \text { months }\end{array}$ \\
\hline
\end{tabular}

Table 5. Implant dimensions (*Most commonly used implant for immediate placement was RP 5x13mm-14/45)

\begin{tabular}{|l|c|c|c|}
\hline Implant Platform & Implant Length & Maxilla & Mandible \\
\hline NP 3.5mm (total number 5) & $11.5 \mathrm{~mm}$ & 3 & 1 \\
\hline & $15 \mathrm{~mm}$ & 0 & 1 \\
\hline RP 4.3mm (total number 5) & $11.5 \mathrm{~mm}$ & 1 & 0 \\
\hline & $13 \mathrm{~mm}$ & 4 & 0 \\
\hline RP 5mm (total number 35) & $8 \mathrm{~mm}$ & 1 & 0 \\
\hline & $10 \mathrm{~mm}$ & 5 & 2 \\
\hline & $11.5 \mathrm{~mm}$ & 5 & 3 \\
\hline & $13 \mathrm{~mm}$ & $14 *$ & 0 \\
\hline & $15 \mathrm{~mm}$ & 3 & 0 \\
\hline & $18 \mathrm{~mm}$ & 2 & 0 \\
\hline
\end{tabular}

months) in this immediate implant placement series. Since final restoration, no biologic or mechanical complications or failures have been noted. Post-implant surgery medium follow-up time was 16 months ( range 4 - 44 months ) from the censored period as at Nov 2012 by observing clinically immobile implant, negative radiographic peri-implant bone lost, no persistent pain or infection and stable gingival complex.

Amongst 45 implants, 27 were immediately temporary restored and loaded with an overall $100 \%$ survive. Contrary, two immediate implants (staged protocol) were failed need redo prior to 3 months. Both the redo implants located at maxilla molar region of same patient whom was a chronic smoker and noncompliant patient. In fact, one of the implant was osseointegrated with excessive cervical threads exposure, so the author decided re-doing it for the best long-term outcome. Finally, the overall survival rate in this cohort became $95.6 \%$ with stable peri-implant health status.

\section{Discussion}

Traditional guidelines suggested 2 to 3 months of socket remodeling following tooth extraction and further 3 to 6 months of load-free healing after implant insertion were needed for optimal osseointegration [9].
Such a long rehabilitation time jeopardize the patient's quality of life especially in the aesthetic demand region. Similarly, the commonest implant replacement occurred in maxilla incisors region (48.9\%) in our case series (Table 2) that would be the chief concern from patient perspective. We may aware the staged immediate implant group in this cohort need to wait for 3.6 months +1.1 months (range 3 weeks to 6 months) for prosthetic loading in contrast to those patient's implant could function and temporized immediately after tooth extraction. The social and economic impact of reduction in number of surgical procedures and in treatment time is unequivocal.

Not until recently, more long-term scientific evidences ${ }^{5}$ were presented to acknowledge the immediate post-extraction implant

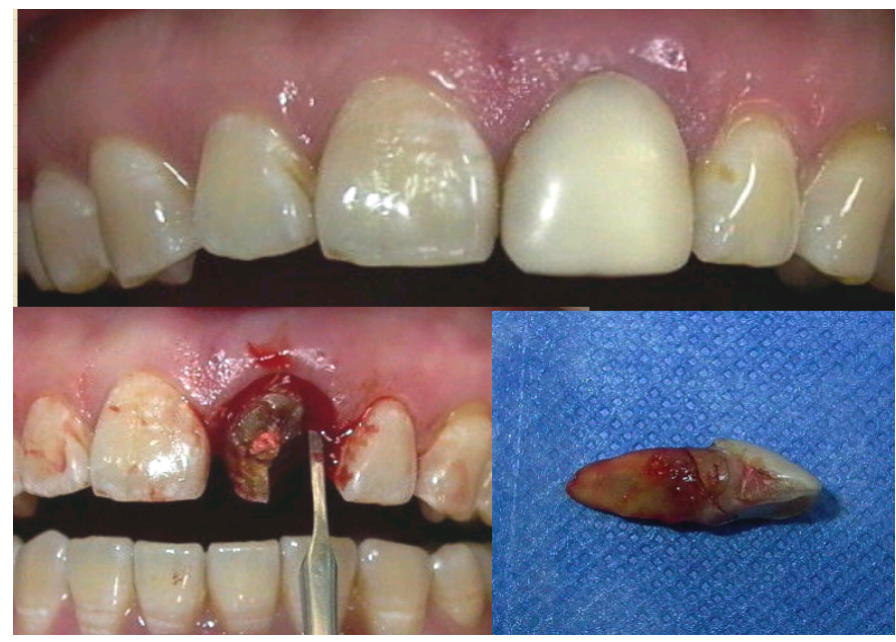

Figure 3. Top: Root treated 21 composite facing crown before extraction, Lower right atraumatic extraction with periotome, Lower left: subgingival \# seen

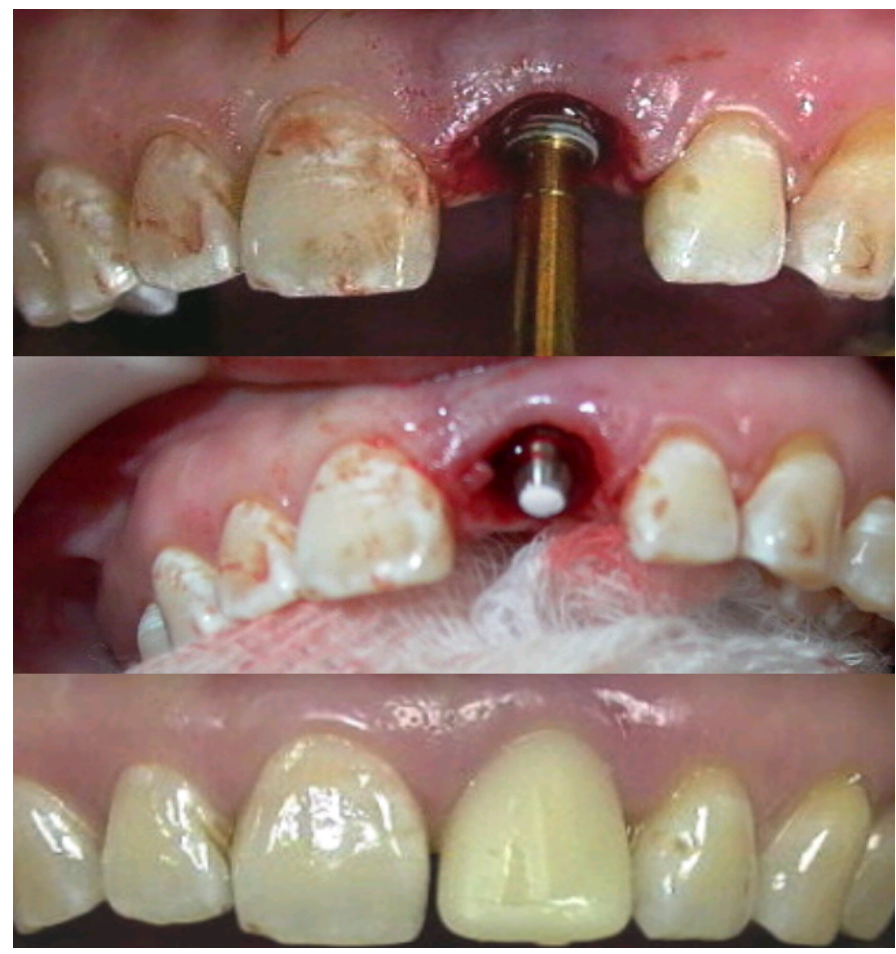

Figure 4. Top: implant insertion into fresh extraction socket, Middle: seating of temporary abutment for immediate temporization, Bottom: temporary crown fitting after 1 month 


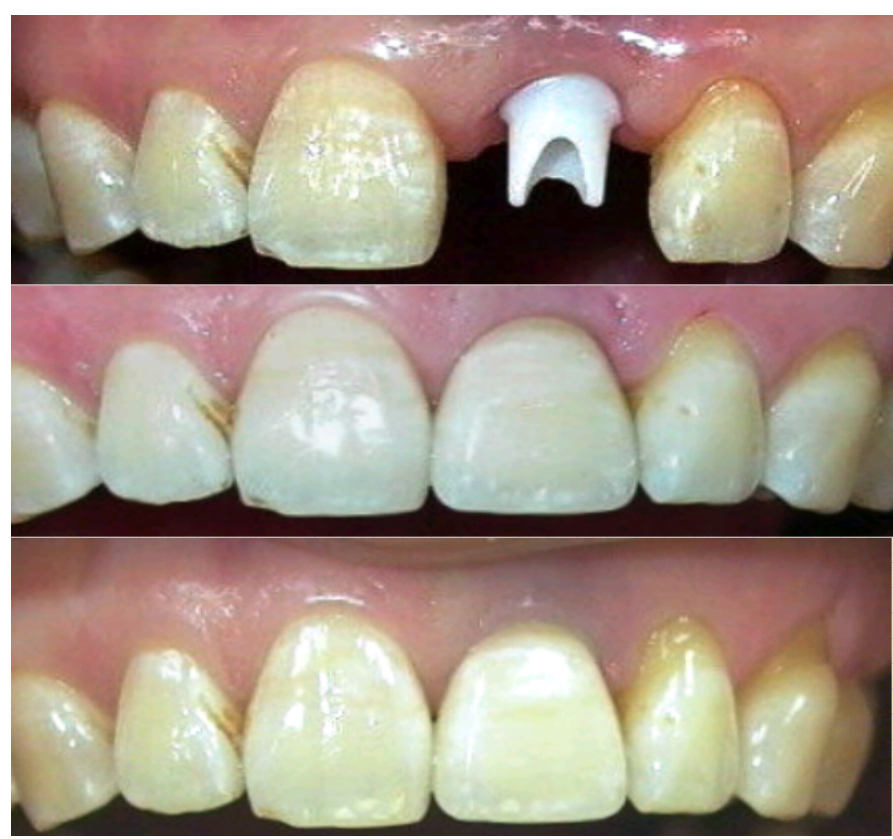

Figure 5. Top: Zirconium abutment seating, Middle: 1-month post-cementation of All Ceramic Crown, Bottom: 2.5 years post-implant, note the gingival margin of 21 crown is very stable even gingival recession noted at 22,23

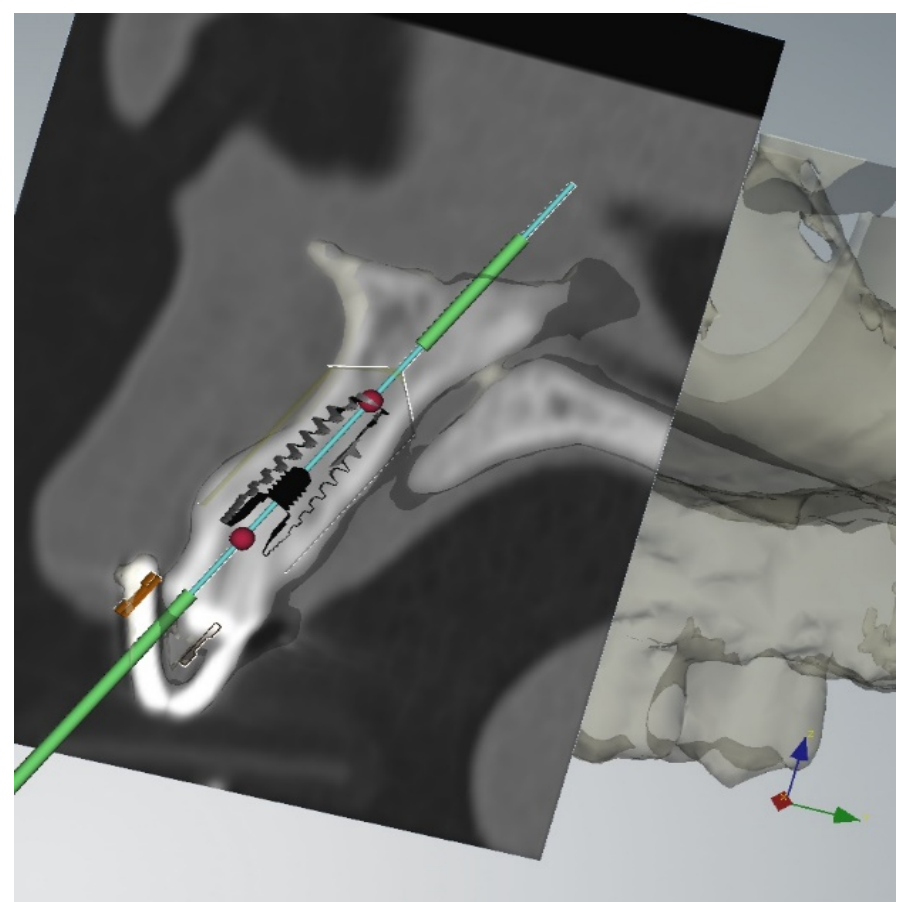

Figure 6. CT pre-op digital planning for implant-socket matching and apical bone engagement anticipation

placement is a predictable implant alternative with a survival rate similar to that of the conventional technique for implant placement. And yet it still categorized as a high risk of implant failure in comparison to delayed implants [10].

Fresh extraction socket as implant recipient site poses a great challenge for the primary stability of implant placement in addition to its continuous physiological remodeling. Following tooth extraction, the biological cascade commenced by alveolar bone resorption both vertically as well as horizontally resulting gingival collapse then followed by complete bone filling of the socket with sign of radioopacity similar to the adjacent bone at 105 days [11]. Schropp, et al. [12] estimated two thirds of the hard and soft tissue changes happen in the first 3 months. In a long-term study [13], the alveolar bone shrinkage could be of $40 \%-60 \%$ in height and width within the first 2 to 3 years. Immediate implant to fresh extraction sites definitely offers alveolar bone maintenance though the concept of alveolar bone preservation is debatable [14]. A more recent animal histomorphological study [15] demonstrated implant immediately placed after tooth extraction fail to preserve the alveolar crest of the socket irrespective of their design and configuration. Even though, bone resorption of the buccal crest is more pronounced when placing large size implant $(5 \mathrm{~mm}$ root-formed implants) when compared to cylindrical implants with a smaller diameter $(3.3 \mathrm{~mm})$. In the same study, the residual defect surrounding the smaller size implant found larger when comparing to the larger size implant group once osseointegration achieved. The necessity of implant-socket gap management fully illustrated by the evidence of $50 \%$ reduction in the original horizontal bone thickness following facial/buccal bone remodeling [16]. Contrary, those with adjunct bone augmentation exhibited a significantly reduced in horizontal resorption of buccal bone [17]. Furthermore, Botticelli, et al. [18] found that $1-1.25 \mathrm{~mm}$ wide peri-implant gap defect healed uneventfully with or without membrane. Therefore, we decided to standardize the adjunct treatment for implant-socket gap $>1.5 \mathrm{~mm}$ whereas $64 \%$ of implant-socket gaps were grafted to aim for a better long term outcome. Interestingly, the demand for GAP grafting were more obvious in wider platform implant $5 \mathrm{~mm}$ (75\%) group; and least in narrow platform implant $3.5 \mathrm{~mm}(20 \%)$ that could be explained by sagittal socket-implant configuration discrepancy.

Insertion torque is most crucial for implant success and survivability especially for immediate loaded single implant. Different implant design could reach its range of insertion torque. Akca, et al. [19] showed the insertion torque values by placing Straumann ${ }^{\mathrm{TM}}$ and Astra Tech ${ }^{\mathrm{TM}}$ implant as high as $57.6 \mathrm{Ncm}$ and $68.5 \mathrm{Ncm}$ respectively at anterior mandible (type I dense cortical bone) whilst the values would drop to an average of $10.7 \mathrm{Ncm}$ and $6.4 \mathrm{Ncm}$ in posterior maxilla (type IV soft bone). The design of NobelActiveTM Implant is a variablethread implant with $1.2 \mathrm{~mm}$ thread spacing which causes the implant to advance $2.4 \mathrm{~mm}$ with each rotation (much higher than conventional implants). Its drilling protocol had been validated according to different bone qualities in addition to its higher insertion torque achieved clinically [7]. Moreover, clinical report [8] showed NobelActive ${ }^{\mathrm{TM}}$ immediate implant placement at the fresh extraction socket (where initial stability is generally compromised) insertion torque could achieve above $50 \mathrm{Ncm}$. In our investigation, similar finding showing $73.3 \%(33 / 45)$ of all implants could achieve insertion torque $>50$ $70 \mathrm{Ncm}$ where the bone quality tends to be medium bone (type II \& III bone) as majority (84.4\%).

When we plan for immediate implant placement in fresh extraction site, several preoperative parameters need sufficient anticipation such as reasons of tooth lost, peri-apical health status and lastly implant size matching. The reason of tooth lost could be a prognostic factor of the implant treatment which truly reflects patient's oral functional status. Such as those patients with advanced periodontal disease (15.6\%) and heavy occlusion that manifested as crack tooth (8.9\%) as the reason of tooth replacement will certainly impose risk to implant maintenance. Most of our patients submitted for immediate implant placement were gone through CT digital planning (Figure 6), implant-socket dimension matching in coronal and apical bony engagement could 
be well anticipated. Yet $5 \mathrm{~mm}$ platform width $13 \mathrm{~mm}$ length implant was the most commonly used implant in this series that account for nearly one third of cases $31.1 \%(14 / 45)$. Peri-apical infection is not a contraindication for immediate implant $[20,21]$. Though occurrence of peri-apical lesion on implants and implant lost showed clear linkage to history of endodontic or peri-apical pathology [22,23]. Adequate pre-operative antibiotic therapy and meticulous local debridement is essential in addition to an adequate insertion torque. In our group of patients, ten teeth $(22.2 \%)$ presented with peri-apical or periodontal abscess prior to surgery that submitted to immediate implant without any case of failure.

Amongst 45 implants, 27 were temporary restored and function immediately. The decision for either immediate temporized and function or in staged procedures were balanced by the patient concern, dental segment location, status of extraction socket and primary stability achieved. We adopted a flapless approach for 26 immediate temporized implants; only 1 immediate molar implant was performed via flap elevation. Flapless approach for implantation is technique demanding and not suitable for gingival contour needs significant labial augmentation. It is always necessary to anticipate labial soft tissue contour stability as the physiological and predictable loss of facial bone might leads to marginal recession of the peri-implant mucosa [24]. With the advent of imaging technology, Cone Beam CT study showed dimensional changes to the peri-implant facial bone in maxillary anterior single immediate implant placement and temporized at both horizontal and vertical area [25]. Though various studies [26,27] showed platform switched implants could achieve a much stable marginal bone level and highly satisfactory esthetic result. The coronal back tapering of NobelActive ${ }^{\mathrm{TM}}$ implant is a build in platform switch design to enhance peri-implant osseous gingival support that should be an optimal design for immediate placement at aesthetic demand region.

Finally, the overall cumulative survival rate of the present study was $95.6 \%$ which is comparable to the reported literatures $[5,20,29]$. Moreover our immediate temporized and function implant achieved $100 \%$ survival. All our patients were advised follow up at 6th months, 1st year, 2nd year after definitive restoration with periodic radiographic monitoring of bone level and peri-implant mucosal status such as stable papilla and soft tissue recession.

\section{Conclusion}

Although immediate implant placement in fresh extraction sites is a challenging implant procedure, observance to a standard protocol could produce a predictable and remarkable cumulative survival rate at $95.6 \%$ showed in this series. As the study was a retrospect patient data in a private clinical setup, we admit the quality of data volume ,patient selection and follow up may not comparable to institutional environment. This report summarized a clinical protocol with literature supported evidences by using a variable-thread, high insertion torque implant (NobelActiveTM) for immediate implant placement in fresh extraction sites listed as followings:

1. Well appraise the reasons of tooth lost, its peri-apical health status and patient compliancy as selection criteria for immediate implant placement especially those for temporized and function immediately;

2. Pre-operative Implant-socket size matching and apical bone quantification by digital Panoramic X-ray and further precision by dental CT is essential;
3. Flapless approach for implant installation at aesthetic demand region. For implant-socket gap $>1.5 \mathrm{~mm}$ need graft augmentation to optimize the long-term peri-implant healing;

4. High insertion torque for initial stability to be achieved $>50-75 \mathrm{Ncm}$;

5. Immediate temporization \& function in the aesthetic demand region could achieve at $100 \%$ success survival rate;

6. Vigilant post-implant monitoring strategy is the corner-stone of success for immediate placement especially for immediate temporized and function cases

\section{Disclaimers}

The authors do not have any financial interests in organizing this clinical study in related to the products listed.

\section{References}

1. Kline R, Hoar JE, Beck GH (2002) A prospective multicenter clinical investigation of a bone quality-based dental implant system. Imp Dent 11: 224-234.

2. Romeo E, Chiapasco M, Ghisolfi M (2002) Long-term clinical effectiveness of oral implants in the treatment of partial edentulism. Seven-year life table analysis of a prospective study with ITI dental implants system used for single-tooth restorations. Clin Oral Implants Res 13: 133-143.

3. Griffin TJ, Cheung WS (2004) The use of short, wide implants in posterior areas with reduced bone height: a retrospective investigation. J Prosthet Dent 92: 139-144. [Crossref]

4. Schulte W, Kleineikenscheidt H, Linder K, Schareyka R (1978) The Tubingen immediate implant in clinical studies. Dtsch Zahnarztl Z 5: 348-359.

5. Enriquez-Sacristan C, Barona-Dorado C, Calvo-Guirado JL, et al. (2001) Immediate post-extraction implants subject to immediate loading: A meta-analytic study. Med Oral Patol Oral Cir Buccal 16: 919-924.

6. Wang HL, Ormianer Z, Palti A (2006) Consensus conference on immediate loading: The single tooth and partial edentulous areas. Implant Dentistry 15: 324-331.

7. Kielbassa Am, Martinez-de Fuentes R, Goldstein M (2009) Randomized controlled trial comparing a variable-thread novel tapered implant: Interim one-year results. J Prosthet Dent 101: 293-305.

8. Irinakis T, Wiebe C (2009) Clinical evaluation of the nobel active implant system: A case series of 107 consecutively placed implants and a review of the implant features. J Oral Implant 35: 283-288

9. Adell R, Lekholm U, Rockler B, Brånemark PI (1981) A 15-year study of osseointegrated implants in the treatment of the edentulous jaw. Int J Oral Surg 10: 387-416. [Crossref]

10. Esposito M, Grusovin MG, Polyzos IP (2010) Timing of Implant placement after tooth extraction: immediate, immediate-delyed or delayed implants? A Cochrane systematic review 3: 189-205.

11. Mangos JG (1941) The healing of extraction wounds: a microscopic and radiographic investigation. New Zealand Dental J 37: 4-23.

12. Schropp L, Wenzel A, Kostopoulos L, Karring T (2003) Bone healing and soft tissue contour changes following single tooth extraction: a clinical and radiographic 12 month prospective study. Int J Perio Restor Dent 23: 313-323.

13. Ashman A (2000) Ridge preservation: important buzzwords in dentistry. Gen Dent 48 304-312. [Crossref]

14. Pagni G, Pellegrini G, Giannobile WV, Rasperini G (2012) Postextraction alveolar ridge preservation: biological basis and treatments. Int J Dent 2012: 151030. [Crossref]

15. Caneva M, Salata LA, deSouza SS (2010) Hard tissue formation adjacent to implants of various size and configuration immediately placed in extraction sockets: an experimental study in dogs. Clin Oral Impl Res 21: 885-890.

16. Botticelli D, Berglundh T, Lindhe J (2004) Hard-tissue alternations following immediate implant placement in extraction sites. J Clin Periodontol 31: 820-828.

17. Chen ST, Darby KB, Reynolds EC (2007) A prospective clinical study of nonsubmerged immediate implants; Clinical outcomes and esthetic results. Clin Oral Implants Res 18: 552-562. 
18. Botticelli D, Berglundh T, Buser D, Lindhe J (2003) The jumping distance revisited: An experimental study in the dog. Clin Oral Implants Res 14: 35-42. [Crossref]

19. Akca K, Chang TL, Tekdemir I, Fanuscu MI (2006) Biomechanical aspects of initial intraosseous stability and implant design. A quantitative micro-morphometric analysis. Clin Oral Impl Res 17: 465-472.

20. McAllister BS, Cherry JE, Kolinski ML (2012) Two-year evaluation of a variablethread tapered implant in extraction sites with immediate temporization: A multicenter clinical trial. Int J Oral \& MaxFac Implants 27: 611-618

21. Novaes Junior AB, Vidigal Junior GM, Novaes AB (1998) Immediate implants placed into infected sites: A histomorphometric study in dogs. Int J Oral Maxillofac Implants 13: $422-427$.

22. Novaes AB Jr, Marcaccini AM, Souza Sl (2003) Immediate placement of implants into periodontally infected sites in dogs. Int J Oral Maxillofac Implants 18: 391398

23. Quiynen M, Gijbels F, Jacobs R (2003) An infected jawbone site compromising successful osseointegration. Periodontol 33: 129-144.
24. Quirynen M, Vogels R, Alsaadi G, Naert I, Jacobs R, et al. (2005) Predisposing conditions for retrograde peri-implantitis, and treatment suggestions. Clin Oral Implants Res 16: 599-608. [Crossref]

25. Chen ST, Darby IG, Reynolds EC (2009) Immediate Implant placement postextraction without flap elevation. J Periodontol 80: 163-172.

26. Roe P, Kan JYK, Rungcharassaeng K (2012) Horizontal and vertical dimensional changes of peri-implant facial bone following immediate placement and provisionalization of maxillary anterior single implants: A 1-year cone beam computed tomography study. Int J Oral Maxillofac Implants 27: 393-400.

27. Vela-Nebot X, Rodriguez-Ciurana X,Rodado-Alonso C (2006) Benefits of an implant platform modification technique to reduce crestal bone resorption. Implant Dent 15 313-320.

28. Lazzara RJ, Porter SS (2006) Platform switching: a new concept in implant dentistry for controlling postrestorative crestal bone levels. Int J Periodontics Restorative Dent 26: 9-17. [Crossref]

29. Quirynen M, Van Assche N, Botticelli D, Berglundh T (2007) How does the timing of implant placement to extraction affect outcome. Int J Oral Maxillofac Implants 22: 203-223.

Copyright: $\odot 2018$ Tak-Kun C. This is an open-access article distributed under the terms of the Creative Commons Attribution License, which permits unrestricted use, distribution, and reproduction in any medium, provided the original author and source are credited. 